\title{
On a Class of Continued Fractions.
}

\author{
By Dr Lester R. Ford.
}

(Read 8th December 1916. Received 20th February 191\%.)

1. The continued fractions treated in this paper are of the general form

$$
s_{0}-\frac{1}{s_{1}}-\frac{1}{s_{2}}-\frac{1}{8_{3}} \ldots
$$

where $s_{0}, s_{1}, s_{2}, \ldots$ are real integers (positive, negative, or zero). An arbitrary real number can, of course, be developed in such a fraction in an infinite variety of ways. The continued fractions discussed here have a number of striking properties and present numerous contrasts with the ordinary continued fraction usually employed.

We shall represent the convergents of the above fraction by

$$
\frac{p_{0}}{q_{0}}, \frac{p_{1}}{q_{1}}, \frac{p_{2}}{q_{2}}, \ldots
$$

Here $p_{0}=1, q_{n}=0, p_{1}=s_{0}, q_{1}=1$, and the numerators and denominators of the following convergents are calculated by means of the recurrence formulae

$$
p_{n+1}=p_{n} s_{n}-p_{n-1}, \quad q_{n+1}=q_{n} s_{n}-q_{n-1} .
$$

We have always

$$
p_{n+1} q_{n}-p_{n} q_{n+1}=-1
$$

\section{Definition of the Continued Fractions.}

The continued fractions of this paper are constructed as follows: Let $r$ and $h$ be any two real positive numbers satisfying the inequalities

$$
h=\frac{1}{2} \sqrt{3}, \quad r \equiv \frac{1}{2} h .
$$


Let $\omega$ be the real number to be developed in a continued fraction.

(a) Take for $s_{0}$ the nearest integer to the quantity

$$
\omega+\sqrt{h(2 r-h)} \text {. }
$$

(b) Knowing the successive convergents $p_{n-1} / q_{n-1}$ and $p_{n} / q_{n}$, where $p_{n} q_{n-1}-p_{n-1} q_{n}=-1$, to find $s_{n}$ and hence $p_{n+1} / q_{n+1}$.

Put

$$
u_{n}=p_{n}-\omega q_{n} .
$$

Let $s_{n}$ be the nearest integer to the quantity

$$
\frac{u_{n-1} \pm \sqrt{h\left(2 r-h u_{n}{ }^{2}\right)}}{u_{n}}
$$

where the sign of the radical is the same as the sign. of $u_{n}$. The following convergent can then be found from the formulae (3).

By means of $(a)$ we are provided with the first two convergents, $1 / 0$ and $s_{0} / 1$, and from these we proceed step by step to construct the continued fraction. It remains to show that the quantities from which $s_{0}, s_{1}, \ldots$ are determined are always real, and secondly that the continued fraction converges to the value $\omega$. These will be established in the following section.

\section{Geometrical Interpretation.}

In the complex $z$-plane $(z=x+i y)$ let a circle $C$, of radius $r$, tangent to the real axis at the point $x=\omega$, and lying in the upper half-plane, be constructed. Construct also the line $y=h$. Since $r \equiv \frac{1}{2} h$, this line intersects $C$. The right-hand intersection has the $x$-coordinate $\omega+\sqrt{h(2 r-h)}$, from which $s_{0}$ is determined.

Now consider the transform of the line $y=h$ by the application of a transformation of the well-known Modular Group

$$
z^{\prime}=\frac{a z+b}{c z+d}, \quad a d-b c=1
$$

where $a, b, c, d$ are integers. If we represent by $\bar{z}$, the conjugate of $z$, i.e. $\bar{z}=x-i y$, we can write the line $y=h$ in the form

$$
z-\bar{z}=2 i h \text {. }
$$

Putting for $z$ its value from $(9), z=\left(-d z^{\prime}+b\right) /\left(c z^{\prime}-a\right)$, and reducing, we get as the transform of $(10)$ the following :-

$$
\left(z^{\prime}-\frac{a}{c}-\frac{i}{2 h c^{2}}\right)\left(\overline{z^{\prime}}-\frac{a}{c}+\frac{i}{2 h c^{2}}\right)=\frac{1}{4 h^{2} c^{3}} \text {. }
$$


This is a circle with centre $\frac{a}{c}+\frac{i}{2 h c^{2}}$ and of radius $\frac{1}{2 h c^{2}}$. It is then tangent to the real axis at the point $x=a / c$, the transform of the point $z=\infty$.

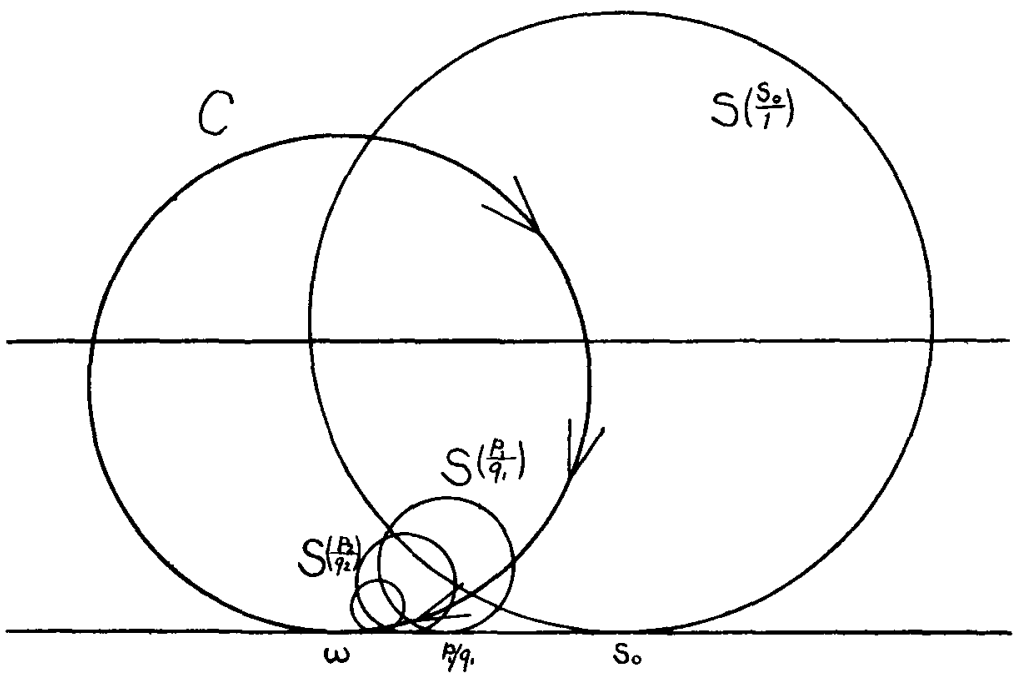

The circle $S(p / q)$. - At each rational point $x=p / q$ of the real axis let us construct a circle tangent to the real axis there, lying in the upper half plane, and having the radius $1 / 2 h q^{2}$ (the fraction $p / q$ being in its lowest terms). We shall call this circle $S(p / q)$. There is a transformation of the type (9) carrying $z=\infty$ to any rational point of the real axis (such that $a / c=p / q$ ); hence we see from (11) that $S(p / q)$ is the transform of $y=h$ by this transformation. We conclude also that a transformation (9) carrying $p / q$ to $p^{\prime} / q^{\prime}$ transforms $S(p / q)$ into $S\left(p^{\prime} / q^{\prime}\right)$; for we could break the transformation into two-one carrying $p / q$ to $\infty$, and $S(p / q)$ into $y=h$, and a second carrying $\infty$ to $p^{\prime} / q^{\prime}$ and $y=h$ into $S\left(p^{\prime} / q^{\prime}\right)$. The line $y=h$ is the $S$-circle of $z=\infty$, which we shall represent by $1 / 0$; and we shall write $y=h$ as $S(1 / 0)$. The part of the plane above the line $S(1 / 0)$ corresponds to the interiors of the other $S$-circles. 
The $S$-circles of the integral points $s / 1$ are of radius $1 / 2 h$. We find easily that $S(8 / 1)$ cuts from the line $y=h$ a segment of length $2 \sqrt{1-h^{2}}$. Since $h=\frac{1}{2} \sqrt{3}$, this length $\equiv 1$. As the points $s / 1$ are a unit distance apart, it follows that every point of the line $S(1 / 0)$ ( $\infty$ excepted) is interior to one at least of the circles $S(8 / 1)$. Transforming $S(1 / 0)$ to $S(p / q)$, we conclude that every point of the circumference $S(p / q)(p / q$ excepted $)$ is interior to at least one other S-circle.

We shall now show that the convergents of our continued fraction have the following property: The circle $S\left(p_{n} / q_{n}\right)$ intersects the circle $C$; and as we pass around $C$ in a clockwise direction, the last intersection with $S\left(p_{n} / q_{n}\right)$ is a point lying within $S\left(p_{n+1} / q_{n+1}\right)$.

The highest point of $C$ lies within $S(1 / 0)$. The $x$-coordinate of the last interection of $C$ with $S(1 / 0)$, viz. $\omega+\sqrt{h(2 r-h)}$, lies by the definition of $s_{0}$ between $s_{0}-\frac{1}{2}$ and $s_{0}+\frac{1}{2}$. This point of intersection then lies within the segment of length $>1$, which $S\left(s_{0} / 1\right)$ cuts from $S(1 / 0)$.

Now let us show that in general the last intersection of $C$ with $S\left(p_{n} / q_{n}\right)$, as we pass round $C$ in a clockwise direction, lies within $S\left(p_{n+1} / q_{n+1}\right)$. Let $p_{n-1} / q_{n-1}$ be the convergent preceding $p_{n} / q_{n}$, and $p_{n} q_{n-1}-p_{n-1} q_{n}=-1$. Making the transformation

$$
z^{\prime}=\frac{q_{n-1} z-p_{n-1}}{q_{n} z-p_{n}},
$$

which is of the Modular Group, $z=p_{n} / q_{n}$ goes to $z=\infty$, and hence $S\left(p_{n} / q_{n}\right)$ becomes $S(1 / 0)$. The circle $C$ becomes a circle $C^{\prime}$ tangent to the real axis at the point $\omega^{\prime}$ where

$$
\omega^{\prime}=\frac{q_{n-1} \omega-p_{n-1}}{q_{n} \omega-p_{n}}=\frac{u_{n-1}}{u_{n}} .
$$

The radius of $C^{\prime}$ is most easily established as follows. If a circle $K$ be drawn tangent to the $x$-axis at $x=p_{n} / q_{n}$ and tangent to $C$, we find by elementary geometry that its radius is $u_{n}{ }^{2} / 4 r q_{n}{ }^{2}$. A reference to (10) and (11) shows that when $p_{n} / q_{n}$ is transformed to $\infty, K^{\prime}$ becomes the line $y=2 r / u_{n}{ }^{2}$. Since $C^{\prime}$ is tangent to this line, its radius is $r / u_{n}^{2}$.

The last intersection of $C$ with $S\left(p_{n} / q_{n}\right)$ becomes the last intersection of $C^{\prime}$ with $y=h$ as we pass round $C^{\prime}$ in a clockwise 
direction; that is, the right hand intersection. This is easily found to be

$$
\omega^{\prime}+\sqrt{2 h r / u_{n}^{2}-h^{2}},
$$

which reduces at once to (8). This point lies within $S\left(s_{n} / 1\right)$ where $s_{n}$ is defined from (8). Now carrying everything back by the inverse of (12), viz. $z=\left(p_{n} z^{\prime}-p_{n-1}\right) /\left(q_{n} z^{\prime}-q_{n-1}\right), s_{n}$ becomes $\left(p_{n} s_{n}-p_{n-1}\right) /\left(q_{n} s_{n}-q_{n-1}\right)$ or $p_{n+1} / q_{n+1}$. Hence $S\left(s_{n} / 1\right)$ becomes $S\left(p_{n+1} / q_{n+1}\right)$, which contains the last point of intersection of $C$ with $S\left(p_{n} / q_{n}\right)$ whieh was to be established.

The expression (14), or (8), being the $x$-coordinate of a real intersection of two circles, is always real.

The convergence of the fraction is easily proved. There is only a finite number of rational numbers $p / q$ in any finite interval of the $x$-axis, whose denominators are less in absolute value than any constant, however large. Since the radius of $S(p / q)$ is inversely proportional to $q^{2}$, there is only a finite number of $S$-circles intersecting $C$ and of radius greater than any positive constant, however small. Hence, as $n$ increases, the radius of $S\left(p_{n} / q_{n}\right)$ approaches 0 , and since $S\left(p_{n} / q_{n}\right)$ intersects $C$, its point of tangency approaches $\omega$. That is,

$$
\operatorname{Lim}_{n=\infty} \frac{p_{n}}{q_{n}}=\omega .
$$

\section{Properties of this class of Continued Fractions.}

In the following sketch of the properties of the fraction and of its convergents we point out in particular its contrasts with the ordinary continued fraction.

(a) The convergents of the ordinary continued fraction are alternately less and greater than the sum $\omega$. The convergents of a continued fraction of this class are greater than $\omega$, with the possible exception of a finite number at the beginning of the suite. For if $p_{n} / q_{n}<\omega, S\left(p_{n} / q_{n}\right)$, which is tangent to the $x$-axis at a point to the left of $\omega$ and contains in its interior a point of the right half of $C$, must have a radius greater than that of $C$. There are clearly only a finite number of such circles possible.

(b) In the ordinary continued fraction $q_{n+1}>q_{n}(n>1)$. In this continued fraction we can have $q_{n+1}<q_{n}$. This fact is illustrated in the second of the examples below. 
(c) The convergents of the ordinary continued fraction satisfy an inequality of the form

$$
\left|\frac{p_{n}}{q_{n}}-\omega\right|<\frac{k}{q_{n}^{2}}, k=1
$$

From the fact that the expression $2 r-h u_{n}{ }^{2}$ of $(8)$ is positive, we have

or

$$
\begin{gathered}
u_{n}{ }^{2}=\left(p_{n}-\omega q_{n}\right)^{2}<2 r / h \\
\left|\frac{p_{n}}{q_{n}}-\omega\right|<\frac{k}{\left|q_{n}\right|}, \quad k=\sqrt{\frac{2 r}{h}} .
\end{gathered}
$$

The inequality thus involves only the first power of $q_{n}$ in the denominator, and it can easily be shown that no inequality of the type (16) can be satisfied by all the convergents, however large $k$ may be. (See the first example below, where $p_{n} / q_{n}-\omega=1 / 3 q_{n}$ ). It is possible to prove that in (17) $k$ can be given the smaller value $\sqrt{r(2 h+1 / 2 h)}$.

(d) If $\omega=p / q$, a rational, the ordinary continued fraction for $\omega$ terminates. For this class, however, the continued fraction is non-terminating in general. For if it terminate, the last convergent must be $p / q$, and $S(p / q)$ must contain a point of $C$-and all of $C$, since the two circles are tangent-on its interior. Then the radius, $1 / 2 h q^{2}$, of $S(p / q)$ must be greater than $r$. This is true for only a finite number of values of $q$, if at all. Since $|q| \equiv 1$, there are no terminating fractions if $r>1 / 2 h$.

(e) Probably the most striking contrast between the two kinds of continued fractions which we are comparing is found in their periodicity. The ordinary continued fraction is periodic (i.e. $s_{n+k}=s_{n}, n>m$ ) if $\omega$ is an irrational root of an equation of the form

$$
A x^{2}+B x+C=0
$$

where $A, B, C$ are integers. The continued fractions of this paper are not periodic if $\omega$ is a quadratic irrational, but they are periodic (excepting the cases in which they terminate) if $\omega$ is rational.

This comes about as follows. The necessary and sufficient condition that a continued fraction of the form (1) be periodic 
is that after a certain point in the suite of convergents $(n>m)$ a relation of the following form should always hold:-

$$
\frac{p_{n+k}}{q_{n+k}}=\frac{a\left(p_{n} / q_{n}\right)+b}{c\left(p_{n} / q_{n}\right)+d}
$$

where $z^{\prime}=(a z+b) /(c z+d)$ is a transformation of the Modular Group, and $k$ is a fixed positive integer. If the fraction converges, its sum $\omega$ is a fixed point of the Modular transformation.

It is well known that if $\omega$ is rational, there are Modular transformations (parabolic in type) with $\omega$ as fixed point, and that for these transformations every circle tangent to the $x$-axis at $x=\omega$ is transformed into itself. The circle $C$ is one of these invariant circles. Now an arc $A$ on $C$ can be chosen such that its transforms by these parabolic transformations cover the whole circumference. Then the finite number of $S$-circles intersecting $A$ is carried by the transformations into all the $S$-circles intersecting $C$. In forming the convergents, which we found to be the coordinates of the points of contact of these $S$-circles with the $x$-axis, we must after a finite number of steps arrive at a convergent derivable from a preceding convergent by one of these parabolic transformations. We can then show without difficulty that the same transformation carries the convergent immediately following the earlier convergent into that immediately following the later one. From this point on a relation of the form (19) is satisfied, and the continued fraction is thus periodic.

We shall not prove that the continued fraction is not periodic if $\omega$ is a quadratic irrational, although it could be done in several ways. We could prove, for example, that a periodic continued fraction of the form (1) has an irrational sum if, and only if, its convergents satisfy an inequality of the type (16) for some value of $k$.

5. Examples.

(a) $\begin{aligned} r=h & =\frac{1}{2}, \omega=\frac{1}{3} \\ \frac{1}{3} & =1-\frac{1}{2}-\frac{1}{3}-\frac{1}{2}-.\end{aligned}$

Convergents : $\frac{1}{0}, \frac{1}{1}, \frac{1}{2}, \frac{2}{5}, \frac{3}{8}, \frac{4}{11}, \frac{5}{14}, \frac{6}{17}, \ldots$ 
(b) If $h$ remain unchanged while $r$ is greatly increased, the continued fruction converges much more slowly. This is obvious geometrically since $C$ then intersects many $S$-circles previously not intersected. For the same reason the period of a periodic fraction is lengthened. Both the long period and the slow convergence appear in the following :-

$$
\begin{gathered}
r=10, h=\frac{1}{2}, \omega=\frac{1}{2} . \\
\frac{1}{2}=4-\frac{1}{1}-\frac{1}{3}-\frac{1}{1}-\frac{1}{5}-\frac{1}{1}-\frac{1}{3}-\frac{1}{2}-\frac{1}{2}-\frac{1}{2}-\frac{1}{1}-\frac{1}{14}-\frac{1}{1}-\frac{1}{2}-\frac{1}{3}- \\
\frac{1}{1}-\frac{1}{4}-\frac{1}{2}-\frac{1}{1}-\frac{1}{7} .
\end{gathered}
$$

Convergents: $\frac{1}{6}, \frac{4}{1}, \frac{3}{1}, \frac{5}{2}, \frac{2}{1}, \frac{5}{3}, \frac{3}{2}, \frac{4}{3}, \frac{5}{4}, \frac{6}{5}, \frac{7}{6}, \frac{1}{1}, \frac{7}{8}, \frac{6}{7}, \frac{5}{8}$, $\frac{9}{11}, \frac{4}{8}, \frac{7}{8}, \frac{10}{13}, \frac{3}{4}, \frac{11}{16}, \frac{8}{11}, \frac{13}{18}, \frac{5}{8}, \frac{12}{17}, \frac{7}{10}, \ldots$ 